\title{
Feedback control of nuclear spin bath of a single hole spin in a quantum dot
}

\author{
Hongliang Pang, ${ }^{1}$ Zhirui Gong, ${ }^{2,1, *}$ and Wang Yao ${ }^{1}$ \\ ${ }^{1}$ Department of Physics, and Center for Theoretical and Computational Physics, The University of Hong Kong, China \\ ${ }^{2}$ School of Physical Sciences and Technology, Shenzhen University, Shenzhen 518060, People's Republic of China \\ (Received 12 July 2014; revised manuscript received 24 December 2014; published 12 January 2015)
}

\begin{abstract}
For a III-V semiconductor quantum dot charged with a single hole, we investigate the feedback control of the nuclear spin bath through dynamical nuclear spin polarization. The scheme utilizes the hole-nuclear flip-flop by their anisotropic hyperfine interaction, where the flip direction of the nuclear spin can be conditioned on the sign of the overall hyperfine field through initialization processes that do not involve explicit measurement. We show that a negative feedback can be implemented to suppress the statistical fluctuations of the nuclear hyperfine field for enhancing the coherence time of the hole spin qubit. Positive feedback can prepare the nuclear spin ensemble into states where the nuclear hyperfine field distribution has two well separated peaks, realizing a quantum heat bath that cannot be described by a single effective temperature.
\end{abstract}

DOI: 10.1103/PhysRevB.91.035305

PACS number(s): 73.21.La, 03.67.Pp, 76.70.Fz

\section{INTRODUCTION}

The spin of a single electron localized in a semiconductor quantum dot has been intensively studied as a carrier of a quantum bit, the elementary building block of a quantum computer [1-5], with potential integrability with the existing semiconductor technology. Compared with silicon quantum dots, the III-V quantum dot has the advantage that the direct band gap of the host material makes possible the ultrafast optical addressability of spin [5]. Optical initialization and readout of single spin, and optically controlled single qubit and two qubit gates have been demonstrated in various III-V quantum dot systems [6-11]. A disadvantage of the III-V quantum dot systems, however, is that all stable isotopes of the group-III and group-V elements have nonzero nuclear spins. Through the contact hyperfine interaction, the electronspin qubit is coupled to $10^{4}-10^{6}$ randomly oriented lattice nuclear spins. The statistical fluctuation of the nuclear spin configurations can result in an electron spin dephasing in the nanosecond time scale, which is the main source of noises for the electron-spin qubit at low temperature [12-21].

This disadvantage of the electron-spin qubit in III-V quantum dots has motivated the study of single hole spin as an alternative. The hole spin qubit can be hosted by the same quantum dot systems that have been investigated for electron spin. For example, for the self-assembled dot embedded in a diode structure, the bias voltage can electrostatically tune the charge configuration of the quantum dot from confining a single electron to a single hole [22-24]. Such hole spin qubit has similar optical controllability as the electron spin [24-27]. The hyperfine interaction between the hole spin and the lattice nuclei is expected to be weaker by about one order of magnitude compared to that of the electron [26,28-30]. Besides, the hole-nuclear coupling is strongly anisotropic: the transverse part is about one order of magnitude smaller than the longitudinal part. The hole spin can then have different decoherence time depending on the quantization axis that is determined by the external magnetic field [25,26]. In the Voigt geometry where the magnetic field is applied along an

*gongzr@hku.hk in-plane direction, it is the weak transverse component of the hole-nuclear coupling that determines the nuclear hyperfine field along the external magnetic field. Thus, in the same nuclear spin environment, the uncertainty in the hole spin Zeeman energy can be about one order of magnitude smaller than that of the electron. A dephasing time of $100 \mathrm{~ns}$ has been measured from the observation of coherent population trapping of a single hole spin in the Voigt geometry [25].

The hyperfine interaction, in the meantime, provides a powerful control to manipulate the nuclear spin environment. A well-known example is the dynamical nuclear spin polarization (DNSP) in which the electron (or hole) spin passes its spin polarization to the lattice nuclei through a flip-flop process [31-40]. The electron-nuclear flip-flop has an energy cost due to the mismatch of the electron and nuclear Zeeman energy in an external magnetic field. In most DNSP control schemes, this energy cost is compensated by emission/absorption of phonons or photons [32,36,41-43]. Alternatively, DNSP can also utilize the oscillating component of the hyperfine coupling when an oscillating electric field induces the electron displacement [44-46]. The energy cost for the electron-nuclear flip-flop can then be directly supplied by the oscillating field. The nuclear spin polarization established by the DNSP may be utilized as a magnetic field to facilitate the control of the electron-spin qubit $[47,48]$. Moreover, various DNSP schemes have also been experimentally and theoretically explored to prepare nuclear bath into states with suppressed statistical fluctuations for enhancing the coherence time of the spin qubit [26,30,44-46,49-56].

In this paper, we explore the feedback control of the nuclear spin bath of a single hole spin. The scheme does not require explicit measurement steps. It utilizes the anisotropic nature of the hole-nuclear interaction. In the absence of external magnetic field, the stronger longitudinal part of the hole-nuclear coupling determines the hole spin eigenstates in the nuclear field, while the weaker transverse part of the coupling (the hole-nuclear flip-flop) causes transition between the eigenstates. By initializing the hole spin in its ground state in the nuclear field, the DNSP can realize a negative feedback that reduces the magnitude of the nuclear field. This can be used to suppress the statistical fluctuations of the nuclear field for enhancing the coherence time of the hole spin qubit. For 
the DNSP, we consider here the one driven by oscillating electric field. Numerical simulations show that the nuclear field fluctuations can be suppressed by an order of magnitude in the millisecond time scale. By initializing the hole spin in its excited state in the nuclear field, the DNSP realizes a positive feedback that amplifies the magnitude of the nuclear field regardless of its initial sign. This can prepare the nuclear spin bath into states where the nuclear field distribution has two well separated peaks, realizing a quantum heat bath that cannot be described by a single effective temperature, providing an ideal model system to investigate the physics that arises from such unconventional quantum heat bath [57-60].

The paper is organized as follows. In Sec. II, we present the feedback control scheme of the nuclear spin bath, and derive the hole-nuclear flip-flop rate in the DNSP driven by the ac electric field. In Sec. III, we numerically investigate the efficiency in the suppression of nuclear field fluctuations by the negative feedback under different choices of the control strategies and parameters. In Sec. IV, we numerically investigate the nuclear state preparation by the positive feedback. Section $\mathrm{V}$ is a brief summary.

\section{FEEDBACK CONTROL OF THE NUCLEAR FIELD BY THE HOLE SPIN}

\section{A. Hole-nuclear hyperfine interaction}

As the hole wave function originates from the atomic $p$ orbital, the contact hyperfine interaction vanishes, leaving only the dipolar hyperfine interaction to couple the hole spin with the lattice nuclei. The wave function of the hole in the quantum dot is mainly contributed by the heavy-hole valence band which has the total spin quantum number along the growth direction $j_{z}= \pm 3 / 2$. The hole-nuclear interaction in the heavy-hole subspace is of the Ising form since the off-diagonal coupling of the hyperfine interaction vanishes between the $j_{z}=3 / 2$ and $j_{z}=-3 / 2$ states [26,30]. In the quantum dot confinement, the hole wave function also has a small contribution from the light-hole valance band because of the heavy- light-hole mixing. Therefore, the hole spin qubit has a finite transverse coupling with the nuclei, which is about one order of magnitude smaller than the longitudinal part. The hyperfine interaction between the single hole spin $\hat{\mathbf{S}}$ and the surrounding nuclear spin bath $\left\{\hat{\mathbf{I}}_{k}\right\}$ reads

$\hat{H}=\sum_{k} \mathcal{A}_{\alpha, h} \frac{c_{0}^{3}}{4}\left|\psi\left(\mathbf{r}_{k}\right)\right|^{2}\left[\hat{S}^{z} I_{k}^{z}+\frac{1}{2} \eta\left(\hat{S}^{+} \hat{I}_{k}^{-}+\hat{S}^{-} \hat{I}_{k}^{+}\right)\right]$,

where $\mathcal{A}_{\alpha, h}$ is the hole hyperfine constant of the material. For In and As, experiments and calculations lead to the estimation $\mathcal{A}_{\alpha, h} \sim 0.1 \mathcal{A}_{\alpha, e}$, where $\mathcal{A}_{A s, e}=46 \mu \mathrm{eV}$ and $\mathcal{A}_{\mathrm{In}, e}=56 \mu \mathrm{eV}$ are the well determined hyperfine constants of the electron $[26,30,61] . \psi\left(\mathbf{r}_{k}\right)$ is the hole envelope wave function at the $k$ th nucleus, and $c_{0}^{3} / 4$ is the volume of each primitive unit cell with $c_{0}$ being the lattice constant.

Similar to the electron-nuclear hyperfine interaction, the dominant part of the hole-nuclear coupling is the short-range one, with the coupling strength being proportional to the hole density at the given nucleus site. The difference is that the hole-nuclear hyperfine interaction is strongly anisotropic. The ratio of the transverse coupling to the longitudinal coupling $\eta$ is proportional to the amount of heavy- light-hole mixing. For a typical self-assembled quantum dot, $\eta \sim 0.1-0.2$ $[9,26]$. In Eq. (1), terms of the order of $\eta^{2}$ and smaller have been dropped [9]. Recent experiments have also suggested that the finite transverse coupling can also arise from the nonzero $d$-shell contribution [62].

Through the hyperfine interaction, the nuclear spins generate an effective magnetic field where the transverse and longitudinal components are respectively $\hat{A}_{x(y)}=$ $\eta \sum_{k} \mathcal{A}_{\alpha, h} c_{0}^{3} / 4\left|\psi\left(\mathbf{r}_{k}\right)\right|^{2} \hat{I}_{k}^{x(y)}$ and $\hat{A}_{z}=\sum_{k} \mathcal{A}_{\alpha, h} c_{0}^{3} / 4\left|\psi\left(\mathbf{r}_{k}\right)\right|^{2} \hat{I}_{k}^{z}$. We define the nuclear field uncertainty $\Delta A_{v} \equiv\left\langle\left(A_{v}-\right.\right.$ $\left.\left.\bar{A}_{v}\right)^{2}\right\rangle^{1 / 2},(v=x, y, z)$, where $\langle\cdots\rangle$ denotes the statistical average over all possible nuclear spin configurations and $\bar{A}_{v}$ is the mean value. Under experimentally achievable temperature, the nuclear spin orientation in a thermal distribution is highly random because of its small energy scale. Thus, the configurations of the nuclear spin bath has large statistical fluctuations, which give rise to a large inhomogeneous broadening of the nuclear field that follows the normal distribution:

$$
f\left(A_{v}\right)=\frac{1}{\pi^{1 / 2} \Delta A_{v}} \exp \left[-\left(\frac{A_{v}}{\Delta A_{\nu}}\right)^{2}\right],
$$

where $\Delta A_{z} \sim \mathcal{A}_{h} / \sqrt{N}$, and $\Delta A_{x(y)} \sim \eta \mathcal{A}_{h} / \sqrt{N}, N$ being the total number of nuclear spins in the quantum dot. In an external magnetic field, the statistical fluctuations of the nuclear field component along the external field direction give rise to pure dephasing of the hole spin qubit in a time scale $T_{2}^{*} \sim(\Delta A)^{-1}$. To have longer coherence time of the qubit, Voigt geometry is therefore more advantageous than the Faraday geometry as $\Delta A_{x(y)} \ll \Delta A_{z}[25]$.

\section{B. Feedback mechanism: Encoding sign of nuclear field to the hole spin state}

In the absence of the external field, the hole spin eigenstates are determined by the $z$ component of the nuclear field $A_{z}$. The hole spin eigenstates are quantized along the $z$ direction, with the sign and magnitude of the Zeeman splitting conditioned on the nuclear spin configuration. The smaller transverse component of the nuclear field can cause a transition between the hole spin eigenstates through the hole-nuclear flip-flop (provided that energy conservation can be satisfied), which changes $A_{z}$. This makes possible the feedback control on the statistical distribution of $A_{z}$.

The basic idea of the feedback control scheme is illustrated in Fig. 1, which consists of two steps. The first step is to polarize the hole spin conditioned on the sign of the nuclear field $A_{z}$, and the second step is to pass the hole spin polarization to the nuclear spin bath through the flip-flop process. The conditional preparation of hole spin polarization can be realized by cooling to the ground state where the spin orientation is determined by the sign of $A_{z}$. If $A_{z}>0\left(A_{z}<0\right)$, the hole spin will then be initialized on the ground state with spin down (up), and the subsequent hole-nuclear flip-flop will pass this spin polarization to the nuclear spin bath by flipping down (up) a nuclear spin through $S^{+} I_{k}^{-}\left(S^{-} I_{k}^{+}\right)$. In either case, the nuclear field magnitude $\left|A_{z}\right|$ will be reduced, which realizes a negative feedback mechanism. This can be utilized to suppress the statistical fluctuations of the nuclear field for enhancing the coherence time of the hole spin qubit. 

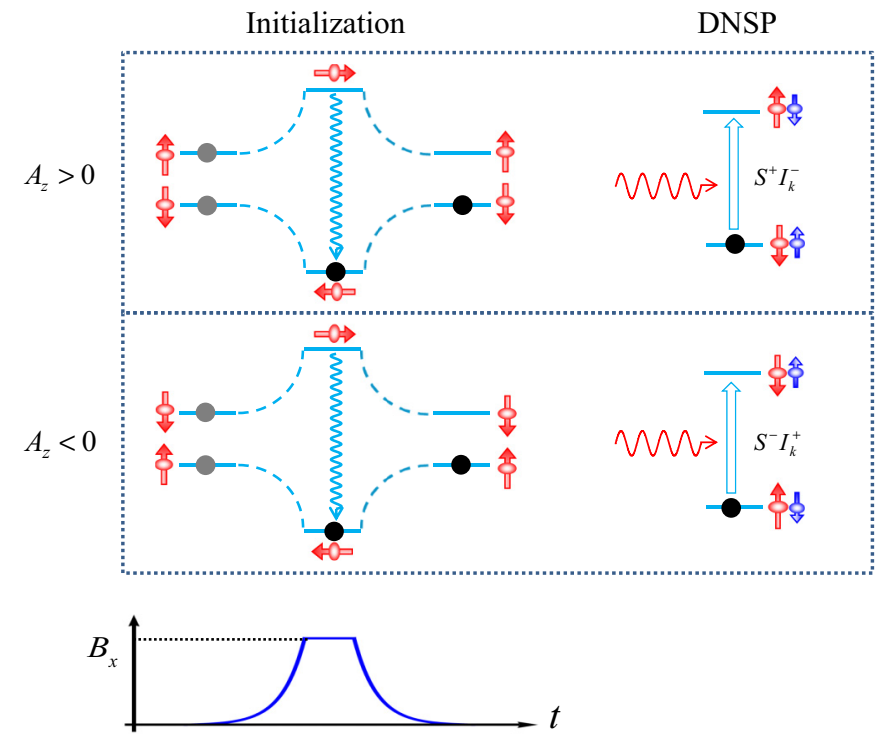

FIG. 1. (Color online) Feedback mechanism for suppressing the nuclear field fluctuation. The hole spin eigenstates (red arrows) are determined by the stronger longitudinal component of the nuclear field $A_{z}$, and the initialization to the ground state correlates the hole spin polarization to the sign of $A_{z}$. To facilitate the initialization, an in-plane magnetic field is adiabatically switched on (see bottom) to induce a sufficiently large Zeeman splitting such that spin relaxation to ground state can be efficient (blue wavy arrows). By adiabatically switching off the magnetic field, the hole spin will remain in the ground state determined by the nuclear field. Here, the black (grey) circles denote the final (initial) population of the hole spin states. This initialization process is followed by a dynamical nuclear spin polarization process to pass the hole spin polarization to the nuclear spin bath (blue arrows), which reduces $\left|A_{z}\right|$ regardless of the initial configuration.

The hole Zeeman splitting in nuclear field is small compared to the thermal energy at typical temperature for the cryogenic experiments [6,7,25-27]. The initialization to the ground state requires an efficient cooling mechanism. We consider the adiabatic switch on of an in-plane external magnetic field to induce a Zeeman splitting larger than the thermal energy. Earlier experiments and theories have shown that the spin qubit relaxation rate increases rapidly with magnetic field [63-65]. Alternatively, with the external magnetic field on, an optical pumping scheme can also be implemented to cool the hole spin to the ground state [5-7], which requires a much smaller magnetic field compared to the phonon-assisted relaxation. It is realized through a Raman-like optical process, consisting of a cw laser pumping one of the transitions in a three-level Lambda system and the spontaneous emission. The three-level Lambda system is formed by the two spin states of the hole and an optical excited state which is a trion. The scheme requires the Zeeman splitting of the hole to be larger than the homogeneous linewidth of the trion in the quantum dot $(\sim 1 \mu \mathrm{eV})$ [66], which can be achieved with a field as small as $10 \mathrm{mT}$. When the external field is adiabatically switched off, the hole spin remains in the ground state in the nuclear field. Ensuring the adiabaticity, we estimate the time scale for switching on and off the external field to be $\sim 100 \mathrm{~ns}$.
When the longitudinal nuclear field strength gets sufficiently suppressed such that it becomes comparable to the transverse nuclear field, the longitudinal and transverse nuclear field components need to be treated on an equal footing. This introduces the limit on using the present description, and a full quantum-mechanical description of both the hole and nuclear spin bath may be necessary to characterize the negative feedback control. It can be expected that the initialization process described above can still establish some correlation between the hole spin polarization with the sign of the longitudinal nuclear field, but the efficiency will be quantitatively affected. We also note that nuclear spins will precess in the external magnetic field. Nevertheless, the effect of such precession can be removed by using two cycles with opposite values of the external field.

\section{Dynamical nuclear spin polarization by the hole}

Once the hole spin is polarized conditioned on the sign of $A_{z}$, this polarization can be passed to the nuclear spin bath through the various dynamical nuclear spin polarization (DNSP) processes [31-46], which will realize the negative feedback to suppress $\Delta A_{z}$. An efficient DNSP mechanism is desired for having a short duration of the feedback control cycle. Here, as an example, we consider the DNSP driven by an oscillating electric field [44-46,52]. In contrast to most DNSP mechanisms in which the energy cost has to be compensated by emission/absorption of phonons or photons, the energy cost is directly supplied by the oscillating electric field, which makes the DNSP efficient.

The hole-nuclear coupling strength is proportional to the hole density at the nucleus site. If an ac electric field is applied to induce a hole displacement $\mathbf{d} \cos \omega t$, an ac term is introduced in the hole-nuclear hyperfine interaction [46],

$$
\hat{H}^{\mathrm{ac}}=\sum_{k} g_{k}\left(\hat{S}^{+} \hat{I}_{k}^{-} e^{-i \omega t}+\hat{S}^{-} \hat{I}_{k}^{+} e^{i \omega t}\right),
$$

where $g_{k} \equiv \eta \mathcal{A}_{\alpha, h} \frac{c_{0}^{3}}{4} \frac{\mathbf{d}}{2} \cdot \nabla\left|\psi\left(\mathbf{r}_{k}\right)\right|^{2}$. If the frequency of the electric field $\omega$ is nearly in resonance with the energy cost for the hole-nuclear flip-flop, DNSP can be efficiently induced. The flip rate of the $k$ th nuclear spin by this ac hyperfine interaction can be estimated using the Fermi's "golden rule" [46],

$$
W_{k}=\left|g_{k}\right|^{2} \frac{\Gamma+\gamma}{\Delta^{2}+(\Gamma+\gamma)^{2}},
$$

where $\Gamma$ is the homogeneous linewidth of the hole spin, and $\gamma$ is the spectral width of the ac electric field. $\Delta$ is the detuning of the central frequency $\omega_{c}$ of the ac electric field from the hole spin Zeeman splitting. The latter corresponds to the energy cost for the hole-nuclear flip-flop. In the absence of external magnetic field, this Zeeman splitting is simply given by $A_{z}$.

For a nuclear spin bath in a thermal distribution, the nuclear field $A_{z}$ has the statistical distribution given by Eq. (2). To squeeze this inhomogeneous broadening, we consider two different scenarios for the DNSP process. The first is a fix-frequency scenario in the presence of a finite magnetic field in the $z$ direction [cf. Fig. 2(a)]. The central frequency $\omega_{c}$ of the ac electric field is set on resonance with the Zeeman splitting $B_{z}$ induced by the external magnetic field. The detuning is then $\Delta=A_{z}$, which is the part of the Zeeman energy due to 
(a)

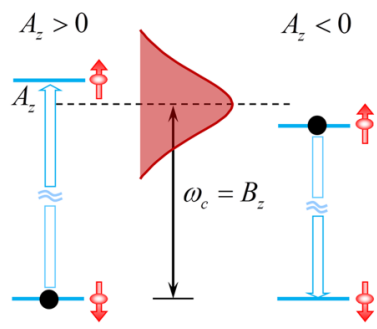

(b)

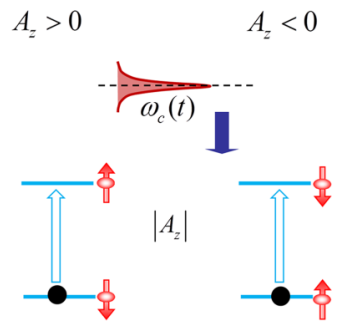

FIG. 2. (Color online) Dynamical nuclear spin polarization driven by ac electric field. (a) The fix-frequency scenario. After the hole spin initialization that correlates hole spin polarization with the sign of $A_{z}$, a magnetic field is turned on in the $z$ direction. The central frequency of the ac electric field $\omega_{c}$ is in resonance with the hole spin Zeeman splitting $B_{z}$ by the external magnetic field. The spectral width of the oscillating electric field is chosen to be comparable with the initial statistical fluctuations of $A_{z}$. (b) The scan-frequency scenario. External magnetic field is absent, and $A_{z}$ determines the hole spin eigenstates. The ac electric field has a narrow spectral width and a scanning central frequency $\omega_{c}(t) . \omega_{c}$ is initially larger than the statistical broadening of $A_{z}$ and is gradually swept down. When the scan is slow enough, $A_{z}$ can adiabatically follow $A_{z}$ towards zero. The black circles denote the hole spin population after the initialization step (cf. Fig. 1).

the nuclear field. From Eq. (4), it is obvious that only those nuclear configurations with $A_{z} \lesssim \gamma$ will be affected by the DNSP. Thus this scenario requires a large $\gamma$ to efficiently squeeze the inhomogeneous broadening. Large $\gamma$, on the other hand, reduces the flip rate and leads to a slow DNSP rate.

The second choice is a scan-frequency scenario in the absence of external magnetic field. The ac electric field initially has a central frequency $\omega_{c}>\Delta A_{z}$ and a small spectral width $\gamma$. The central frequency is then swept down along with the DNSP. In such case, $\Delta=\omega_{c}-\left|A_{z}\right|$. Regardless of the initial nuclear configuration, the DNSP will take effect once $\omega_{c}$ comes into resonance with $\left|A_{z}\right|$. If the frequency scanning speed $\dot{\omega}_{c}$ is slow enough, by the negative feedback control, $\left|A_{z}\right|$ will simply follow $\omega_{c}$ until a sufficiently small broadening is achieved.

\section{Probability of a hole-nuclear flip-flop event}

A nuclear spin flip in the DNSP is always accompanied by the flip of the hole spin, after which the hole spin state need to be initialized again to start a new cycle of feedback control. Here we want to find out the probability to have a hole-nuclear flip-flop event as a function of the duration of the DNSP driving, so as to determine the proper duration of the DNSP process in each cycle.

Equation (4) gives the rate to flip the $k$ th nuclear spin, which is applicable only at the short-time limit. The overall probability for a hole-nuclear flip-flop event can be tracked from the dynamics of the hole spin. The hole spin couples collectively with all nuclear spins in the bath. Assuming the initial state of the nuclear spin bath and the hole spin is $\left|\Psi_{i}\right\rangle=|\psi\rangle_{n} \otimes|\uparrow\rangle_{h}$, the ac hyperfine interaction couples it to the final state $\left|\Psi_{f}\right\rangle=\sum_{k} g_{k} \hat{I}_{k}^{+}|\psi\rangle_{n} \otimes|\downarrow\rangle_{h}$, with the effective
Rabi frequency,

$$
\Omega=\frac{\left\langle\Psi_{f}\left|\hat{H}^{a c}\right| \Psi_{i}\right\rangle}{\left\langle\Psi_{f} \mid \Psi_{f}\right\rangle} \sim \sqrt{\sum_{k}\left|g_{k}\right|^{2}} .
$$

Under the resonance condition, i.e., $\Delta=0$, the steady state of the reduced hole spin density matrix will be an equal mixture of the spin up and spin down states in the presence of hole spin decoherence and the finite linewidth of the ac electric field. The time scale to approach such steady state can be taken as the duration of the ac driven DNSP, to optimize the efficiency of each feedback cycle.

The effective dynamics of the hole spin in the DNSP can then be calculated using the master equation

$$
\dot{\rho}=-i\left[\Delta \hat{S}^{z}+\left(\Omega \hat{S}^{+}+\text {c.c. }\right), \rho\right]+\mathcal{L}[\rho],
$$

where $\rho$ is the density matrix of the hole spin, and $\mathcal{L}[\rho]$ is the Lindblad term describing the hole spin decoherence and the finite spectral width of the ac driving field. Under the resonance condition (i.e., $\Delta=0$ ), the transition probability is defined as $P(t)=\langle\downarrow|\rho| \downarrow\rangle$, and we find

$$
P(t)=\frac{1}{2}\left[1-\frac{1}{\left(\gamma_{+}-\gamma_{-}\right)}\left(e^{-(1 / 2) t \gamma_{-}} \gamma_{+}-e^{-(1 / 2) t \gamma_{+}} \gamma_{-}\right)\right],
$$

where $\gamma_{ \pm}=\Gamma+\gamma \pm \sqrt{(\Gamma+\gamma)^{2}-\Omega^{2}}$. The probability of hole-nuclear flip-flop event saturates at 0.5 in the long-time limit. Under the overdamped condition $(\Omega<\Gamma+\gamma)$, the time scale for $P(t)$ to approach this steady-state value is $(\Gamma+\gamma) / \Omega^{2}$, which can be set as the duration $t_{c}$ of the ac electric field. In our numerical simulation, by balancing (a)

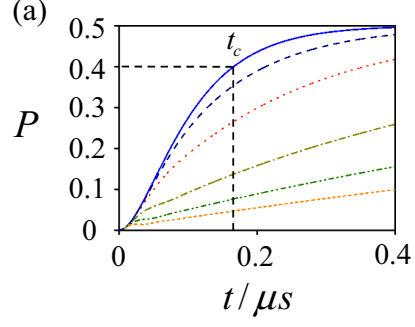

(b)

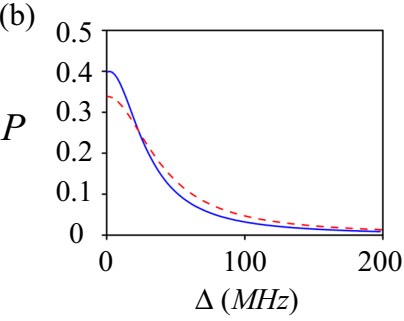

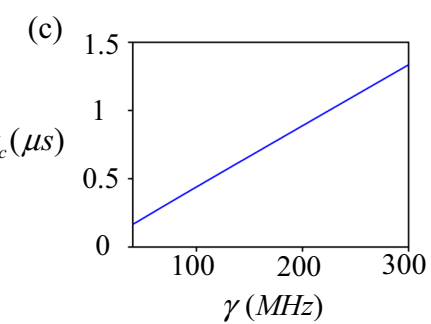

FIG. 3. (Color online) (a) The hole-nuclear flip-flop probability $P$ as a function of time under the DNSP by the ac electric field. The blue solid, navy dashed, red dotted, dark yellow dash-dotted, olive dash-dot-dotted, and orange short dashed curves correspond respectively to the detuning value $\Delta \equiv A_{z}-\omega_{c}=0,20,40,60,80$, $100 \mathrm{MHz} . \Omega=9.5 \mathrm{MHz}$ and $\gamma=40 \mathrm{MHz}$. We define $t_{\mathrm{c}}$ to be the time when $P\left(t_{c}\right)=0.4$ at zero detuning. (b) Solid curve: $P$ as a function of detuning at the cut-off time $t_{\mathrm{c}}$ shown in (a). Dashed line is the plot of the normalized Fermi's golden-rule rate for comparison [cf. Eq. (4) in main text]. (c) $t_{\mathrm{c}}$ as a function of $\gamma$, which has a linear dependence for the overdamped condition $(\Omega<\Gamma+\gamma)$ considered here. 
between the flip-flop probability and the time, we choose $t_{c}$ such that $P\left(t_{c}\right)=0.4$ for the resonant configurations with $\Delta=0$. With the duration of the ac driving determined, the flip-flop probability in the presence of finite $\Delta$ can also be calculated, which is of the Lorentz shape (cf. Fig. 3).

We note that in the ac electric field, the hole spin flip can also be induced by the spin-orbit coupling (SOC). The treatment here has not taken this into account. In the presence of SOC, the feedback control scheme can remain the same, but the SOC will affect the feedback control efficiency. For short duration of the ac driving, the hole spin flip rate will be the sum of those due to the SOC and due to the hyperfine interaction respectively. The hole spin flip by the SOC then corresponds to a "null" cycle in the feedback control of nuclear spin bath, so the overall feedback control efficiency is determined by the ratio between the hyperfine mediated hole spin flip rate to the SOC mediated hole spin flip rate. For long duration of the ac driving, the relation between the hole spin reduced density matrix and the transfer of polarization to nuclear spins can be complicated due to the interplay of the SOC and the hole-nuclear hyperfine interaction, and the treatment here does not work when the SOC is strong.

\section{NUMERICAL SIMULATION ON THE SQUEEZING OF NUCLEAR FIELD STATISTICAL FLUCTUATIONS}

In this section, we numerically study the feedback control to squeeze the nuclear field statistical fluctuations under various choices of control scenarios and parameters. As an example, we consider the self-assembled InAs quantum dots. The relevant hyperfine constants in Eq. (1) are taken to be $\mathcal{A}_{\mathrm{As}, h}=$ $4.6 \mu \mathrm{eV}$ and $\mathcal{A}_{\text {In }, h}=5.6 \mu \mathrm{eV}$. Without loss of generality, we model the quantum dot by a $16 \mathrm{~nm} \times 16 \mathrm{~nm} \times 4 \mathrm{~nm}$ rectangular box, where the envelope wave function of the hole is

$$
\psi\left(\mathbf{r}_{k}\right)=\sqrt{\frac{8}{a b c}} \cos \left(\frac{\pi}{a} x\right) \cos \left(\frac{\pi}{b} y\right) \cos \left(\frac{\pi}{c} z\right)
$$

with $a=b=32 \mathrm{~nm}$ and $c=8 \mathrm{~nm}$. The lattice constant $c_{0}=$ $6.058 \AA$. Under thermal equilibrium at typical temperature for the cryogenic experiments [6,7,25-27] (i.e., the initial state of the nuclear spin bath), the nuclear field distribution has a full width at half maximum (FWHM) of $2 \Delta A_{z} \sim 100 \mathrm{MHz}$.

In the simulations, the initial nuclear spin configuration is randomly generated and the subsequent evolution is given by the Monte Carlo method. For each feedback control cycle, the probability $P$ for the hole-nuclear flip-flop event to occur is given in Sec. II D, which depends on the DNSP pumping duration as well as the detuning $\Delta$ between the instantaneous value of hole spin Zeeman energy and $\omega_{c}$. We set the duration $t_{c}$ of DNSP in each cycle such that $P\left(t_{c}\right)=0.4$ for $\Delta=0$. The time scale of hole spin initialization is short compared to $t_{c}$, hence the overall period of the control cycle is just given by $t_{c}$. Given a hole-nuclear flip-flop in a control cycle, a quantum jump occurs in the nuclear spin bath $|\psi\rangle \rightarrow \hat{I}_{k}^{-}|\psi\rangle(|\psi\rangle \rightarrow$ $\left.\hat{I}_{k}^{+}|\psi\rangle\right)$ if $A_{z}$ is positive (negative). The conditional probability (branching ratio) that the jump is on the $k$ th nuclear spin is $\left|g_{k}\right|^{2} /\left(\sum_{k}\left|g_{k}\right|^{2}\right)$. In all simulations in this section, we assume that ac electric field induces a root-mean-square displacement
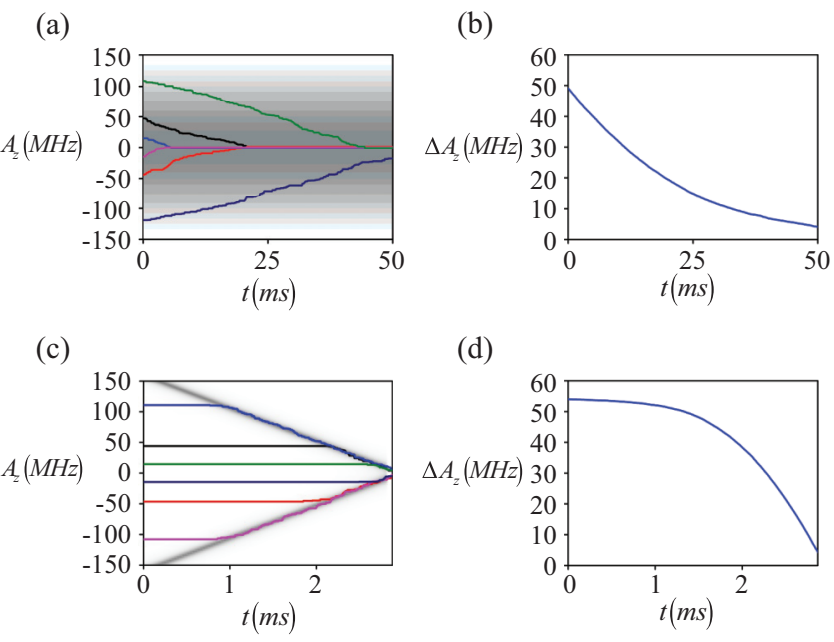

(d)

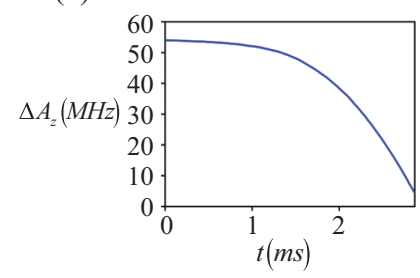

(e)

(f)
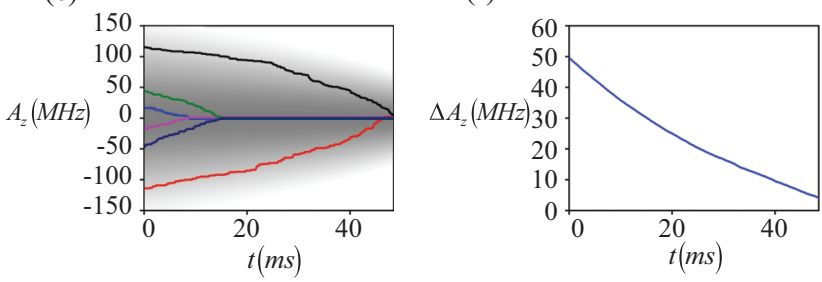

FIG. 4. (Color online) Nuclear field evolution under the negative feedback control. Panels (a), (c), and (e) show the trajectories for a few representative initial nuclear spin configurations, and (b), (d), and (f) show the evolution of the the nuclear field inhomogeneous broadening $\Delta A_{z}$. Panels (a) and (b) use the fix-frequency scenario [see text and Fig. 2(a)], where the ac electric field has a central frequency in resonance with the Zeeman splitting by external magnetic field $\left(\omega_{c}=\right.$ $B_{z}$ ). The spectral width of the ac field is also fixed: $\gamma=150 \mathrm{MHz}$. Panels (c) and (d) use the scan-frequency scenario in absence of external magnetic field [cf. Fig. 2(b)], where the spectral width of the ac field is fixed at $\gamma=4 \mathrm{MHz}$. The central frequency scans with the speed $\dot{\omega}_{c}=-53.3 \mathrm{MHz} / \mathrm{ms}$. Panels (e) and (f) also use the fix-frequency scenario in presence of magnetic field, but the spectrum width varies with time. The spectral distribution of the ac field (shifted by the value of $B_{z}$ ) is indicated by the shaded area. $\Omega=1 \mathrm{MHz}$.

of $0.1 \mathrm{~nm}$ of the hole, and the resultant value of $\Omega$ is about $1 \mathrm{MHz}$.

Figures 4(a) and 4(b) uses the fix-frequency scenario of DNSP in a finite external magnetic field along the $z$ direction [cf. Fig. 2(a)]. A large spectral width of the ac electric field is used: $\gamma=150 \mathrm{MHz}$, comparable to the FWHM of the initial nuclear field distribution. The duration of the ac driving DNSP is determined to be $t_{c}=60.3 \mu \mathrm{s}$. Figure 4(a) gives the trajectories for a few representative initial configurations of the nuclear spin bath, and the shadow indicates the spectral distribution of the ac electric field. Figure 4(b) is the plot of the corresponding change of the nuclear field fluctuation $\Delta A_{z}$ as a function of time. $\Delta A_{z}$ can be squeezed by about one order of magnitude in a time of $50 \mathrm{~ms}$. With such wide spectral width of the ac electric field, the DNSP rate is almost the same under all possible nuclear configurations [cf. Eq. (4)], hence the nuclear field changes with a nearly constant speed [Fig. 4(a)]. The large spectral width, on the other hand, requires 
a long duration of each DNSP, limiting the time efficiency of the control.

Figures 4(c) and 4(d) use the scan-frequency scenario of DNSP in the absence of magnetic field. The spectral width of the ac electric field is fixed at $\gamma=4 \mathrm{MHz}$. The duration of the ac driving DNSP is then substantially shorter, $t_{c}=1.5 \mu \mathrm{s}$. The central frequency of the ac electric field is $\omega_{c}=160 \mathrm{MHz}$ initially, and scans down with the speed $\dot{\omega}_{c}=-53.3 \mathrm{MHz} / \mathrm{ms}$. Figure 4(c) gives the trajectories for a few representative initial configurations of the nuclear spin bath, and the shadow indicates the spectral distribution of the ac electric field. Obviously, the configurations will have nonzero transition probability only when $A_{z}$ falls into the shadow region (i.e., becomes close in resonance with $\omega_{c}$ ). Figure $4(\mathrm{~d})$ is the plot of the corresponding change of the nuclear field fluctuation $\Delta A_{z}$ as a function of time. $\Delta A_{z}$ can be squeezed by about one order of magnitude in a time of $3 \mathrm{~ms}$, much shorter than the fix-frequency scenario in Figs. 4(a) and 4(b).

Figures 4(e) and 4(f) also use the fix-frequency scenario under finite magnetic field. The difference from Figs. 4(a) and 4(b) is that the spectral width of the ac electric field is reduced along with the squeezing of $\Delta A_{z}$. Figure 4(e) gives the trajectories for a few representative initial configurations of the nuclear spin bath, and Fig. 4(f) is the plot of the corresponding change of the nuclear field fluctuation $\Delta A_{z}$ as a function of time. The temporal change of $\gamma$ is illustrated by the narrowing of the shadow region in Fig. 4(e). Smaller $\gamma$ leads to reduced duration $t_{c}$ of the DNSP pumping [cf. Eq. (4)], and consequently the nuclear field evolves faster. The nuclear field statistical fluctuation $\Delta A_{z}$ is squeezed by about one order of magnitude in a time of $50 \mathrm{~ms}$. The overall time efficiency is improved compared to the simulation in Figs. 4(a) and 4(b).

Obviously, the scan-frequency scenario has better time efficiency compared to the fix-frequency scenario. For the scan speed used in Figs. 4(c) and 4(d), the nuclear field value simply follows the scanning frequency towards zero. This implies that a faster scan might improve the time efficiency. We examine here the effect of frequency scan speed on the overall efficiency of the feedback control. Figure 5 shows the evolution of the nuclear field distribution $f\left(A_{z}\right)$ as a function of time, under two different choices of the scan speed $\dot{\omega}_{c}=50 \mathrm{MHz} / \mathrm{ms}$ and $\dot{\omega}_{c}=100 \mathrm{MHz} / \mathrm{ms}$ respectively. In both cases, the spectral width of the ac driving field is fixed as $\gamma=4 \mathrm{MHz}$. Clearly, with the faster scan, a faster squeezing is achieved initially. However, the nuclear field eventually fails to follow the frequency scan, leaving behind a fairly wide distribution. Therefore $\Delta A_{z}$ saturates at a larger value and the degree of squeezing is worse compared to the slow scan case. A proper scan speed can be chosen to balance between the time efficiency and the degree of squeezing.

\section{POSITIVE FEEDBACK CONTROL FOR PREPARING BIMODAL DISTRIBUTION OF NUCLEAR FIELD}

The key for the feedback control is to correlate the hole spin polarization with the instantaneous sign of the nuclear field through the initialization in the adiabatically switched in-plane magnetic field (cf. Fig. 1). By adding a $\pi$ pulse to flip the hole spin polarization after its initialization, the same control can also realize a positive feedback. As shown in Fig. 6,
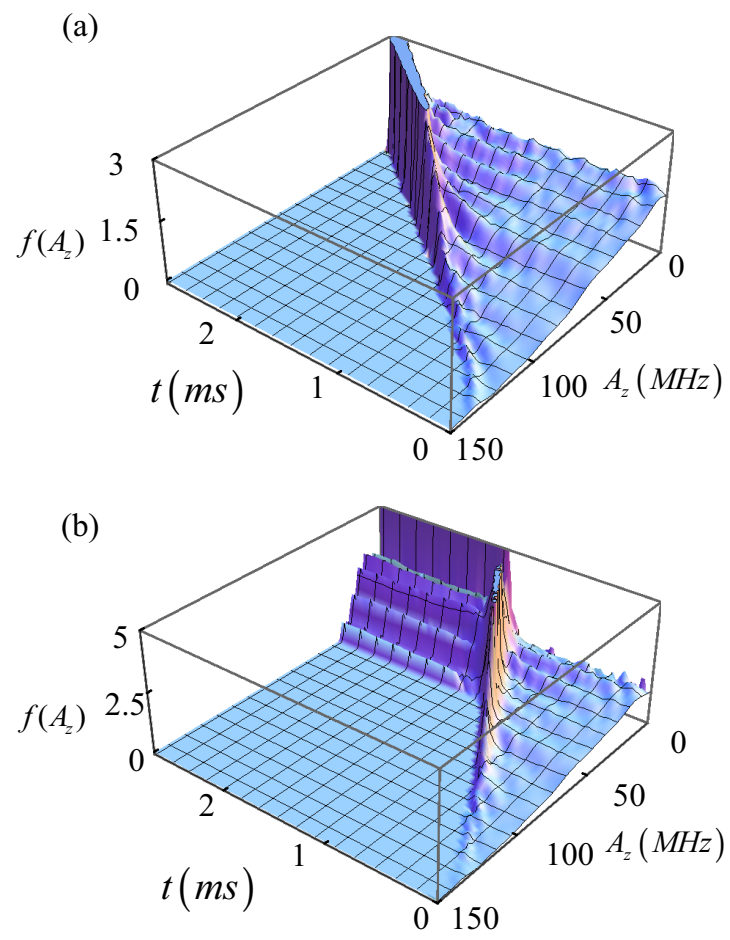

FIG. 5. (Color online) The evolution of the nuclear field distribution $f\left(A_{z}\right)$ under the negative feedback control with the scanfrequency scenario. The spectral width of the ac electric field is fixed at $\gamma=4 \mathrm{MHz}$, and at $t=0$ the central frequency is $\omega_{c}=140 \mathrm{MHz}$. (a) $\dot{\omega}_{c}=50 \mathrm{MHz} / \mathrm{ms}$ and (b) $\dot{\omega}_{c}=100 \mathrm{MHz} / \mathrm{ms}$.

if $A_{z}>0\left(A_{z}<0\right)$, the hole spin is polarized on the excited state with spin up (down) after the $\pi$ pulse, and the subsequent hole-nuclear flip-flop will pass this spin polarization to the nuclear spin bath by flipping up (down) a nuclear spin through $S^{-} I_{k}^{+}\left(S^{+} I_{k}^{-}\right)$. This will amplify the magnitude of the nuclear field, regardless of its sign, which will expectedly prepare the nuclear field distribution $f\left(A_{z}\right)$ into a bimodal one.

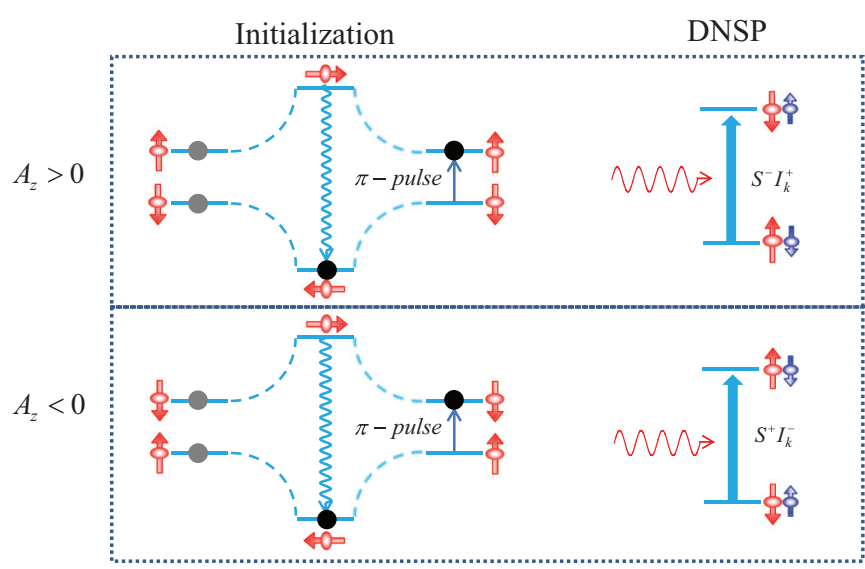

FIG. 6. (Color online) Schematic illustration of the positive feedback control. The initialization of the hole spin (red arrows) is similar to that in the negative feedback control, except that following the spin relaxation to the ground state, a $\pi$ pulse flips the spin to the excited state. The DNSP control is identical to that in the negative feedback control. 
(a)

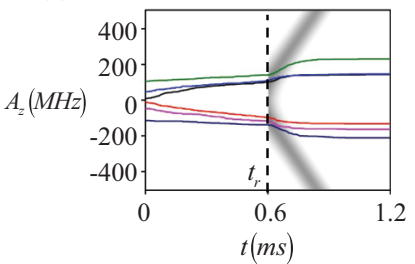

(c)

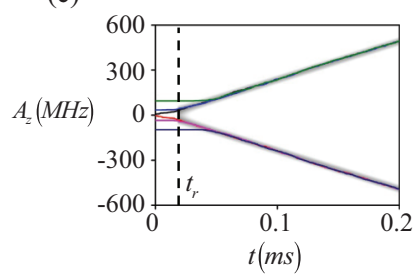

(b)

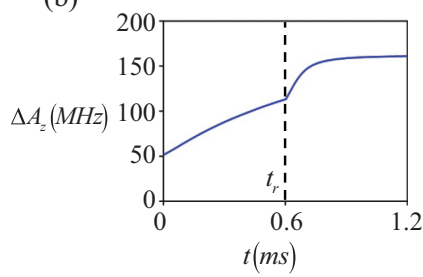

(d)

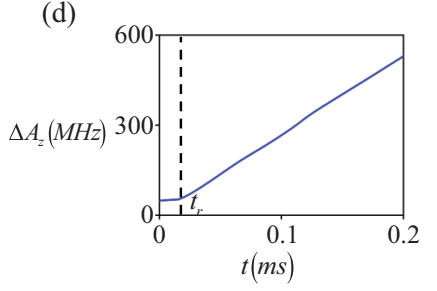

FIG. 7. (Color online) (a),(c) The trajectories for a few representative initial configurations of the nuclear spin bath and (b),(d) plot of the corresponding change of the nuclear field broadening $\Delta A_{z}$ as a function of time for various choices of the ac field spectral width $\gamma$ and frequency scanning speed $\dot{\omega}_{c}$. The frequency of the electric field is fixed as $\Omega=9.5 \mathrm{MHz}$. The spectral width and frequency scanning speed are $\gamma=150 \mathrm{MHz}, \dot{\omega}_{c}=1.67 \mathrm{MHz} / \mu$ s for (a) and (b) and $\gamma=40 \mathrm{MHz}, \dot{\omega}_{c}=3 \mathrm{MHz} / \mu$ s for (c) and (d). $\Omega=9.5 \mathrm{MHz}$, which corresponds to the root-mean-square displacement of $1 \mathrm{~nm}$ of the hole.

We consider here the combination of the fix-frequency scenario and the scan-frequency one. As discussed in the previous section, the scan-frequency scenario has much better time efficiency since nuclear field will simply follow the scan of the central frequency of the ac electric field. However, as the central frequency of the ac field has to be finite in practice, those nuclear configurations with small nuclear field magnitude cannot be efficiently affected in the scan-frequency DNSP. Thus, the fix-frequency scenario of DNSP is used at the initial stage, which can amplify the nuclear field magnitude regardless of its initial value, with a low time efficiency though. The fix-frequency DNSP can eventually deplete the nuclear field distribution in a window of width $\gamma$ centered at zero, after which we switch to the scan-frequency scenario of DNSP for further amplification of $\left|A_{z}\right|$.

The numerical simulations for the positive feedback control are presented in Fig. 7. The quantum dot is the same as the one studied in the previous section. Figures 7(a) and 7(b) use ac electric field with a spectrum width $\gamma=150 \mathrm{MHz}$, comparable to the initial nuclear field broadening at thermal distribution. Figure 7(a) shows the trajectories for a few representative initial configurations of the nuclear spin bath. For $0<t<$ $t_{r}$, the fix-frequency scenario is adopted for the DNSP, and $t>t_{r}$ is the scan-frequency control with scan speed $\dot{\omega}_{c}=$ $1.67 \mathrm{MHz} / \mu$ s. Figure 7(b) plots the nuclear field uncertainty $\Delta A_{z}$. We note that $\Delta A_{z}$ here is determined largely by the separation of the two peaks in the bimodal distribution.

Figures 7(c) and 7(d) use ac electric field with a narrower spectral width $\gamma=40 \mathrm{MHz}$, and at $t=t_{r}$ the control switches to the scan-frequency one with scan speed $\dot{\omega}_{c} \approx 3 \mathrm{MHz} / \mu \mathrm{s}$. The shadow region represents the scanning of the frequency distribution of the ac electric field. With this set of control
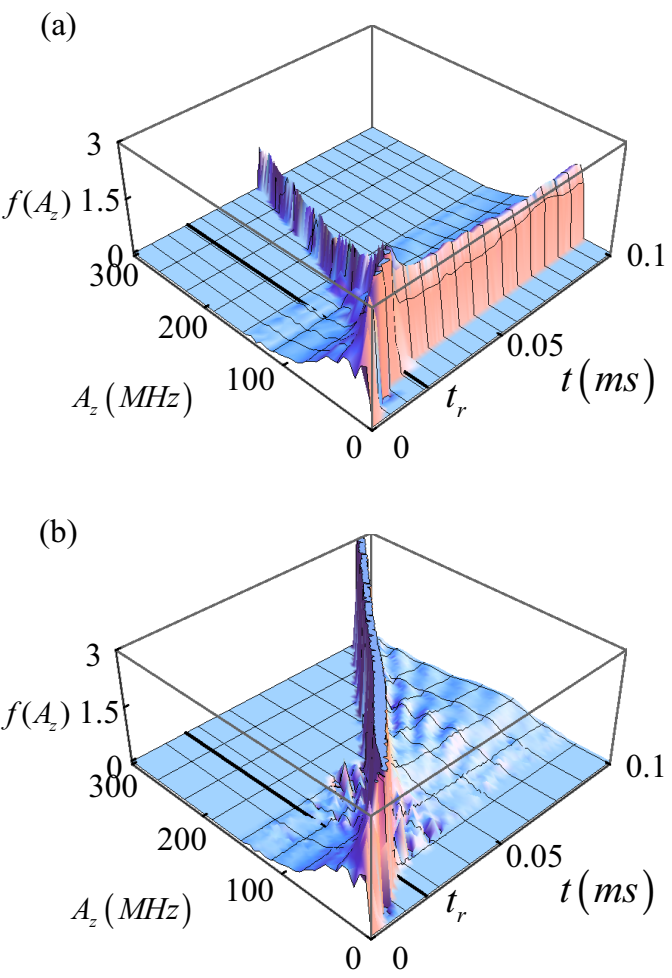

FIG. 8. (Color online) The evolution of the nuclear field distribution $f\left(A_{z}\right)$ under the positive feedback control. The spectral width of the ac electric field is fixed at $\gamma=40 \mathrm{MHz}$. The scanning speed is $\dot{\omega}_{c}=10 \mathrm{MHz} / \mu \mathrm{s}$ in (a), and $\dot{\omega}_{c}=3 \mathrm{MHz} / \mu \mathrm{s}$ in (b). At $t=t_{r}$, the control switches from the fix-frequency scenario to the scan-frequency one.

parameters, we find that the nuclear field magnitude simply follows the frequency scan of the ac electric field. This allows sufficient amplification of the nuclear field magnitude. The nuclear field distribution segregates into two peaks, each with a very narrow width. Figure $7(\mathrm{~d})$ is a plot of the corresponding change of the nuclear field uncertainty $\Delta A_{z}$, which measures the separation of the two peaks rather than the width of each. As the nuclear field follows the frequency scan, a linear increment of $\Delta A_{z}$ is seen here, as opposed to the saturation behavior in Figs. 7(a) and 7(b).

Figure 8 shows the evolution of the nuclear field distribution $f\left(A_{z}\right)$, comparing two different scan speeds. For the faster scan $\left(\dot{\omega}_{c}=10 \mathrm{MHz} / \mu \mathrm{s}\right)$ shown in Fig. 8(a), we can see that only a small portion of the nuclear configurations can follow the frequency scan of the ac electric field when the scan begins at $t_{r}=0.02 \mathrm{~ms}$. The majority is left over, and the nuclear field distribution has two large broad peaks around $A_{z} \sim \gamma$ and $A_{z} \sim-\gamma$, and two small narrow peaks at $A_{z}=\omega_{c}$ and $A_{z}=-\omega_{c}$. In contrast, for the slower scan $\left(\dot{\omega}_{c}=3 \mathrm{MHz} / \mu \mathrm{s}\right)$ shown in Fig. 8(b), most nuclear configurations follow the scanning frequency of the ac electric field. The initial single broad peak in the nuclear field distribution segregates into two narrow peaks centered at $A_{z}=\omega_{c}$ and $A_{z}=-\omega_{c}$ respectively.

The simulations in Figs. 7 and 8 also imply that the positive feedback can be utilized to prepare various nuclear field distributions that cannot be described by a single temperature. This provides a viable way to engineer a multitemperature 


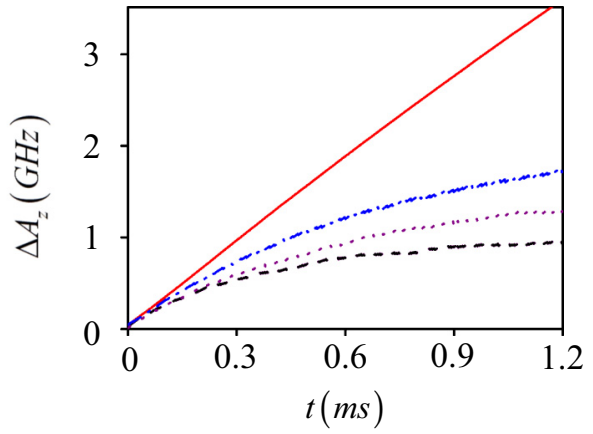

FIG. 9. (Color online) The evolution of the $\Delta A_{z}$ under positive feedback control in the presence of nuclear spin relaxation. The scanning-frequency scenario is used with parameters $\gamma=40 \mathrm{MHz}$, $\dot{\omega}_{c}=3 \mathrm{MHz} / \mu$ s and $\Omega=9.5 \mathrm{MHz}$. The nuclear spin-relaxation time $T_{1}=\infty, 1,0.7,0.5 \mathrm{~ms}$ for the for red solid, blue dash-dotted, purple dotted, and black dashed curves, respectively.

spin bath for investigating the novel physics that can arise from such an unconventional environment [57-60]. Moreover, the bimodal distribution with narrow peak width [cf. Fig. 8(b)] also suggests the possibility of realizing the catlike state of the nuclear spin bath.

Finally, we investigate the effect of nuclear spin relaxation which can compete with the feedback control and affect the control efficiency. The relaxation of the nuclear spins is deleterious to the preparation processes of both the negative and positive feedback schemes because it flips nuclear spin randomly. The nuclear spin-relaxation effect can be more prominent in the positive feedback control where the nuclear field has larger magnitude in the steady state, compared to that in the negative feedback control. Figure 9 shows the evolution of $\Delta A_{z}$ for the positive feedback scheme under different nuclear spin-relaxation time. The parameters are $\gamma=40 \mathrm{MHz}$ and $\Omega=9.5 \mathrm{MHz}$. We find that the effect of nuclear spin relaxation becomes visible when nuclear spin $T_{1} \sim$ ms. For $T_{1} \gg \mathrm{ms}$, nuclear spin relaxation has negligible effects in the duration of the feedback control.

We note that in the negative feedback control, the nuclear spin relaxation is also a competing mechanism by inducing the spectral diffusion, and it can be another factor to limit the maximum suppression of nuclear field statistical fluctuation unless nuclear spin $T_{1}$ is much longer than the time scale of the control.

In the self-assembled quantum dots, the nuclear quadrupolar interaction can introduce uneven spacing of the nuclear energy levels [36,37,40,59,67-70]. Its interplay with the hyperfine interaction leads to nuclear spin depolarization, which can compete with the feedback control. However, experimental and theoretical studies have shown that the depolarization time scale is much slower $(\sim 0.1-1 \mathrm{~s})[36,40,67]$, compared to the time scale of the feedback control in our study, and the effect is therefore negligible.

\section{CONCLUSION}

We have shown that the feedback controls of the nuclear spin bath can be realized, utilizing the anisotropic hyperfine interaction between the nuclear spins with a single hole spin. In the absence of external magnetic field, the stronger longitudinal part of the hole-nuclear coupling determines the hole spin eigenstates. By initializing the hole spin in its ground state in the longitudinal nuclear field, the hole spin polarization can be correlated with the sign of the instantaneous nuclear field. The weaker transverse part of the hole-nuclear coupling can cause the hole-nuclear flip-flop, which passes the hole spin polarization to the nuclear spin bath through the DNSP process. The initialization of hole spin and the DNSP together realize the feedback control of the nuclear hyperfine field. The negative feedback control always reduces the magnitude of the nuclear field, hence squeezing the statistical fluctuations. The positive feedback magnifies the magnitude of nuclear field regardless of its sign, driving the nuclear field distribution into bimodal distribution.

The key for the feedback control lies in the initialization stage where the hole spin polarization is correlated with the instantaneous nuclear field value. In general, the DNSP can be realized with various schemes. Here we considered as example the DNSP driven by ac electric field, and the fix-frequency and scan-frequency scenarios are compared under different choices of the spectral width and scanning speed of the central frequency for the ac field. Numerical simulations show that in the negative feedback control, the nuclear field fluctuations can be suppressed about one order of magnitude within a preparation time of a few ms. Under the positive feedback control, the nuclear field distribution can evolve into two well separated narrow peaks. In such a case, the nuclear spin bath cannot be described by a single effective temperature, which could provide an ideal model system to investigate the interesting physics that arises from such unconventional quantum heat bath [57-60].

\section{ACKNOWLEDGMENTS}

The work was supported by the General Research Fund and Collaborative Research Fund from the Research Grant Council of Hong Kong, the University of Hong Kong under OYRA, and the Croucher Foundation under the Croucher Innovation Award.
[1] D. Heiss, S. Schaeck, H. Huebl, M. Bichler, G. Abstreiter, J. J. Finley, D. V. Bulaev, and D. Loss, Phys. Rev. B 76, 241306(R) (2007).

[2] Semiconductor Spintronics and Quantum Computing, edited by D. D. Awschalom, D. Loss, and N. Samarth (Springer, Berlin, 2002).
[3] D. Loss and D. P. DiVincenzo, Phys. Rev. A 57, 120 (1998).

[4] R. Hanson, L. P. Kouwenhoven, J. R. Petta, S. Tarucha, and L. M. K. Vandersypen, Rev. Mod. Phys. 79, 1217 (2007).

[5] R. B. Liu, W. Yao, and L. J. Sham, Adv. Phys. 59, 703 (2010).

[6] M. Atatüre, J. Dreiser, A. Badolato, A. Högele, K. Karrai, and A. Imamoglu, Science 312, 551 (2006). 
[7] X. Xu, Y. Wu, B. Sun, Q. Huang, J. Cheng, D. G. Steel, A. S. Bracker, D. Gammon, C. Emary, and L. J. Sham, Phys. Rev. Lett. 99, 097401 (2007).

[8] M. Scheibner, M. Yakes, A. S. Bracker, I. V. Ponomarev, M. F. Doty, C. S. Hellberg, L. J. Whitman, T. L. Reinecke, and D. Gammon, Nat. Phys. 4, 291 (2008).

[9] X. Xu, W. Yao, B. Sun, D. G. Steel, A. S. Bracker, D. Gammon, and L. J. Sham, Nature (London) 459, 1105 (2009).

[10] J. Berezovsky, M. H. Mikkelsen, N. G. Stoltz, L. A. Coldren, and D. D. Awschalom, Science 320, 349 (2008).

[11] D. Press, T. D. Ladd, B. Zhang, and Y. Yamamoto, Nature (London) 456, 218 (2008).

[12] I. A. Merkulov, A. L. Efros, and M. Rosen, Phys. Rev. B 65 , 205309 (2002).

[13] S. Saikin and L. Fedichkin, Phys. Rev. B 67, 161302(R) (2003).

[14] S. Saykin, D. Mozyrsky, and V. Privman, Nano Lett. 2, 651 (2002).

[15] Y. Y. Wang and M. W. Wu, Phys. Rev. B 77, 125323 (2008).

[16] A. V. Khaetskii, D. Loss, and L. Glazman, Phys. Rev. Lett. 88, 186802 (2002).

[17] W. A. Coish and D. Loss, Phys. Rev. B 70, 195340 (2004).

[18] C. Deng and X. Hu, Phys. Rev. B 73, 241303(R) (2006).

[19] W. M. Witzel, R. de Sousa, and S. Das Sarma, Phys. Rev. B 72, 161306(R) (2005)

[20] L. Cywinski, W. M. Witzel, and S. Das Sarma, Phys. Rev. Lett. 102, 057601 (2009)

[21] R. de Sousa and S. Das Sarma, Phys. Rev. B 68, 115322 (2003).

[22] M. E. Ware, E. A. Stinaff, D. Gammon, M. F. Doty, A. S. Bracker, D. Gershoni, V. L. Korenev, Ş. C. Bădescu, Y. LyandaGeller, and T. L. Reinecke, Phys. Rev. Lett. 95, 177403 (2005).

[23] E. A. Stinaff, M. Scheibner, A. S. Bracker, I. V. Ponomarev, V. L. Korenev, M. E. Ware, M. F. Doty, T. L. Reinecke, and D. Gammon, Science 311, 636 (2006).

[24] S. E. Economou, J. I. Climente, A. Badolato, A. S. Bracker, D. Gammon, and M. F. Doty, Phys. Rev. B 86, 085319 (2012).

[25] D. Brunner, B. D. Gerardot, P. A. Dalgarno, G. Wüst, K. Karrai, N. G. Stoltz, P. M. Petroff, and R. J. Warburton, Science 325, 70 (2009).

[26] B. Eble, C. Testelin, P. Desfonds, F. Bernardot, A. Balocchi, T. Amand, A. Miard, A. Lemaitre, X. Marie, and M. Chamarro, Phys. Rev. Lett. 102, 146601 (2009).

[27] B. D. Gerardot, D. Brunner, P. A. Dalgarno, P. Öhberg, S. Seidl, M. Kroner, K. Karrai, N. G. Stoltz, P. M. Petroff, and R. J. Warburton, Nature (London) 451, 441 (2008).

[28] P. Fallahi, S. T. Yilmaz, and A. Imamoglu, Phys. Rev. Lett. 105, 257402 (2010).

[29] E. A. Chekhovich, A. B. Krysa, M. S. Skolnick, and A. I. Tartakovskii, Phys. Rev. Lett. 106, 027402 (2011).

[30] J. Fischer, W. A. Coish, D. V. Bulaev, and D. Loss, Phys. Rev. B 78, 155329 (2008).

[31] F. H. L. Koppens, C. Buizert, K. J. Tielrooij, I. T. Vink, K. C. Nowack, T. Meunier, L. P. Kouwenhoven, and L. M. K. Vandersypen, Nature (London) 442, 766 (2006).

[32] A. S. Bracker, E. A. Stinaff, D. Gammon, M. E. Ware, J. G. Tischler, A. Shabaev, Al. L. Efros, D. Park, D. Gershoni, V. L. Korenev, and I. A. Merkulov, Phys. Rev. Lett. 94, 047402 (2005).

[33] D. J. Reilly, J. M. Taylor, J. R. Petta, C. M. Marcus, M. P. Hanson, and A. C. Gossard, Science 321, 817 (2008).
[34] C. W. Lai, P. Maletinsky, A. Badolato, and A. Imamoglu, Phys. Rev. Lett. 96, 167403 (2006).

[35] P.-F. Braun, X. Marie, L. Lombez, B. Urbaszek, T. Amand, P. Renucci, V. K. Kalevich, K. V. Kavokin, O. Krebs, P. Voisin, and Y. Masumoto, Phys. Rev. Lett. 94, 116601 (2005).

[36] A. I. Tartakovskii, T. Wright, A. Russell, V. I. Fal'ko, A. B. Van'kov, J. Skiba-Szymanska, I. Drouzas, R. S. Kolodka, M. S. Skolnick, P. W. Fry, A. Tahraoui, H.-Y. Liu, and M. Hopkinson, Phys. Rev. Lett. 98, 026806 (2007).

[37] K. Ono and S. Tarucha, Phys. Rev. Lett. 92, 256803 (2004).

[38] K. R. Wald, L. P. Kouwenhoven, P. L. McEuen, N. C. van der Vaart, and C. T. Foxon, Phys. Rev. Lett. 73, 1011 (1994).

[39] H. Y. Yu, Y. Luo, and W. Yao, Chin. Phys. Lett. 30, 077302 (2013).

[40] P. Maletinsky, A. Badolato, and A. Imamoglu, Phys. Rev. Lett. 99, 056804 (2007).

[41] J. Danon and Y. V. Nazarov, Phys. Rev. Lett. 100, 056603 (2008).

[42] V. L. Korenev, Phys. Rev. Lett. 99, 256405 (2007).

[43] B. Urbaszek, P.-F. Braun, T. Amand, O. Krebs, T. Belhadj, A. Lemaitre, P. Voisin, and X. Marie, Phys. Rev. B 76, 201301(R) (2007).

[44] E. A. Laird, C. Barthel, E. I. Rashba, C. M. Marcus, M. P. Hanson, and A. C. Gossard, Phys. Rev. Lett. 99, 246601 (2007).

[45] M. S. Rudner and L. S. Levitov, Phys. Rev. Lett. 99, 246602 (2007).

[46] E. I. Rashba, Phys. Rev. B 78, 195302 (2008).

[47] S. Foletti, H. Bluhm, D. Mahalu, V. Umansky, and A. Yacoby, Nat. Phys. 5, 903 (2009).

[48] S. G. Carter, S. E. Economou, A. Greilich, E. Barnes, T. M. Sweeney, A. S. Bracker, and D. Gammon, Phys. Rev. B 89 , 075316 (2014).

[49] I. T. Vink, K. C. Nowack, F. H. L. Koppens, J. Danon, Y. V. Nazarov, and L. M. K. Vandersypen, Nat. Phys. 5, 764 (2009).

[50] E. A. Chekhovich, M. N. Makhonin, K. V. Kavokin, A. B. Krysa, M. S. Skolnick, and A. I. Tartakovskii, Phys. Rev. Lett. 104, 066804 (2010)

[51] E. B. Hale and R. L. Mieher, Phys. Rev. 184, 739 (1969).

[52] W. Yao, Phys. Rev. B 83, 201308(R) (2011).

[53] W. Yang and L. J. Sham, Phys. Rev. B 85, 235319 (2012).

[54] W. Yang and L. J. Sham, Phys. Rev. B 88, 235304 (2013).

[55] C. Latta, A. Högele, Y. Zhao, A. N. Vamivakas, P. Maletinsky, M. Kroner, J. Dreiser, I. Carusotto, A. Badolato, D. Schuh, W. Wegscheider, M. Atature, and A. Imamoglu, Nat. Phys. 5, 758 (2009).

[56] S. E. Economou and E. Barnes, Phys. Rev. B 89, 165301 (2014).

[57] Q. T. Zhuang and B. Wu, Laser Phys. Lett. 11, 085501 (2014).

[58] C.-Y. Cai, S.-W. Li, X.-F. Liu, and C. P. Sun, arXiv:1407.2004.

[59] P. Maletinsky, M. Kroner, and A. Imamoglu, Nat. Phys. 5, 407 (2009).

[60] B. Urbaszek, X. Marie, T. Amand, O. Krebs, P. Voisin, P. Maletinsky, A. Högele, and A. Imamoglu, Rev. Mod. Phys. 85 , 79 (2013)

[61] C. Testelin, F. Bernardot, B. Eble, and M. Chamarro, Phys. Rev. B 79, 195440 (2009).

[62] E. A. Chekhovich, M. M. Glazov, A. B. Krysa, M. Hopkinson, P. Senellart, A. Lemaitre, M. S. Skolnick, and A. I. Tartakovskii, Nat. Phys. 9, 74 (2013).

[63] A. V. Khaetskii and Y. V. Nazarov, Phys. Rev. B 64, 125316 (2001). 
[64] V. N. Golovach, A. Khaetskii, and D. Loss, Phys. Rev. Lett. 93, 016601 (2004).

[65] M. Kroutvar, Y. Ducommun, D. Heiss, M. Bichler, D. Schuh, G. Abstreiter, and J. J. Finley, Nature (London) 432, 81 (2004).

[66] X. Xu, B. Sun, E. D. Kim, K. Smirl, P. R. Berman, D. G. Steel, A. S. Bracker, D. Gammon, and L. J. Sham, Phys. Rev. Lett. 101, 227401 (2008).
[67] C.-W. Huang and X. Hu, Phys. Rev. B 81, 205304 (2010).

[68] J. Ishihara, M. Ono, G. Sato, S. Matsuzaka, Y. Ohno, and H. Ohno, Jpn. J. Appl. Phys. 50, 04DM03 (2011).

[69] N. A. Sinitsyn, Y. Li, S. A. Crooker, A. Saxena, and D. L. Smith, Phys. Rev. Lett. 109, 166605 (2012).

[70] E. A. Chekhovich, M. Hopkinson, M. S. Skolnick, and A. I. Tartakovskii, arXiv:1403.1510. 